\title{
In Vitro and In Vivo Evaluation of Three Newly Isolated Bacteriophage Candidates, phiEF7H, phiEF14H1, phiEF19G, for Treatment of Enterococcus faecalis Endophthalmitis
}

\author{
Tatsuma Kishimoto ${ }^{1}$ (D), Waka Ishida ${ }^{1}$ (D), Tadahiro Nasukawa ${ }^{2}$, Takako Ujihara ${ }^{3}$, Isana Nakajima ${ }^{1}$, \\ Takashi Suzuki ${ }^{4}$, Jumpei Uchiyama ${ }^{2}$ D, Daisuke Todokoro ${ }^{5}$, Masanori Daibata ${ }^{6}$, Atsuki Fukushima ${ }^{1}$, \\ Shigenobu Matsuzaki ${ }^{7}$ and Ken Fukuda ${ }^{1, *}$
}

1 Department of Ophthalmology and Visual Science, Kochi Medical School, Kochi University, Kochi 783-8505, Japan; t.kishimoto@kochi-u.ac.jp (T.K.); wakai@kochi-u.ac.jp (W.I.); jm-i-nakajima@kochi-u.ac.jp (I.N.); asp0322@icloud.com (A.F.)

2 Laboratory of Veterinary Microbiology I, School of Veterinary Medicine, Azabu University, Kanagawa 252-5201, Japan; dv1804@azabu-u.ac.jp (T.N.); uchiyama@azabu-u.ac.jp (J.U.)

3 Science Research Center, Kochi University, Kochi 783-8505, Japan; jm-ujiharat@kochi-u.ac.jp

4 Department of Ophthalmology, Toho University, Tokyo 143-8541, Japan; takashisuzuki58@gmail.com

5 Department of Ophthalmology, Graduate School of Medicine, Gunma University, Gunma 371-8511, Japan; dtodokor@gunma-u.ac.jp

check for updates

Citation: Kishimoto, T.; Ishida, W.; Nasukawa, T.; Ujihara, T.; Nakajima, I.; Suzuki, T.; Uchiyama, J.; Todokoro, D.; Daibata, M.; Fukushima, A.; et al. In Vitro and In Vivo Evaluation of Three Newly Isolated Bacteriophage Candidates, phiEF7H, phiEF14H1, phiEF19G, for Treatment of Enterococcus faecalis Endophthalmitis. Microorganisms 2021, 9, 212. https://doi.org/10.3390/ microorganisms 9020212

Academic Editor: Marco Maria D'Andrea

Received: 29 December 2020

Accepted: 17 January 2021

Published: 20 January 2021

Publisher's Note: MDPI stays neutral with regard to jurisdictional claims in published maps and institutional affiliations.

Copyright: (c) 2021 by the authors. Licensee MDPI, Basel, Switzerland. This article is an open access article distributed under the terms and conditions of the Creative Commons Attribution (CC BY) license (https:/ / creativecommons.org/licenses/by/ $4.0 /)$.
6 Department of Microbiology and Infection, Kochi Medical School, Kochi University, Kochi 783-8505, Japan; daibatam@kochi-u.ac.jp

7 Department of Medical Laboratory Science, Faculty of Health Sciences, Kochi Gakuen University, Kochi 780-0955, Japan; matuzaki@kochi-u.ac.jp

* Correspondence: k.fukuda@kochi-u.ac.jp; Tel.: +81-88880-2391

\begin{abstract}
Post-operative endophthalmitis caused by Enterococcus spp. progresses rapidly and often results in substantial and irreversible vision loss. Therefore, novel alternative treatments that are effective against enterococcal endophthalmitis are required. Bacteriophage therapy has the potential to be an optional therapy for infectious diseases. Therefore, we investigated the therapeutic potential of three newly isolated enterococcal phages, phiEF7H, phiEF14H1, and phiEF19G, in E. faecalisinduced endophthalmitis. These phages could lyse the broad-range E. faecalis, including strains derived from endophthalmitis and vancomycin-resistant E. faecalis in vitro, as determined by the streak test. Morphological and genomic analyses revealed that these phages were classified into the Herelleviridae genus Kochikohdavirus. The whole genomes of these phages contained 143,399, 143,280, and $143,400 \mathrm{bp}$, respectively. Endophthalmitis was induced in mice by injection of three strains of $E$. faecalis derived from post-operative endophthalmitis or vancomycin-resistant strains into the vitreous body. The number of viable bacteria and infiltration of neutrophils in the eye were both decreased by intravitreous injection of phiEF7H, phiEF14H1, and phiEF19G $6 \mathrm{~h}$ after injection of all E. faecalis strains. Thus, these results suggest that these newly isolated phages may serve as promising candidates for phage therapy against endophthalmitis.
\end{abstract}

Keywords: Enterococcus faecalis; bacteriophage; endophthalmitis; Herelleviridae; mouse model; phage therapy

\section{Introduction}

Bacteriophages (phages) are viruses that infect bacteria. Phages can be isolated from environments, including sewage, food, soil, and the gastrointestinal tract. Phages infect bacteria and lyse the cell wall by producing lytic enzymes in the bacteria. Phage therapy is a method using phages or their products as bioagents for the treatment of bacterial infectious diseases. Phages have the potential to be antibacterial agents to solve the problems caused by antimicrobial-resistant bacteria [1]. The therapeutic effects of systemic and topical phage therapy have been previously demonstrated in a mouse model of sepsis or endophthalmitis [2-4]. 
Postoperative endophthalmitis is a severe complication of ocular surgery or intravitreal injection, which can lead to substantially reduced visual acuity or even blindness. The number of postoperative endophthalmitis cases has recently increased in association with an increase in the number of ocular surgeries and intravitreal injection [5,6]. Postoperative endophthalmitis is often caused by Gram-positive bacteria such as enterococci and staphylococci [7]. In particular, enterococcal endophthalmitis has a poorer visual outcome than that caused by Staphylococcus aureus and coagulase-negative Staphylococcus spp. [8]. Prompt diagnosis and appropriate treatment of endophthalmitis are necessary to prevent retinal damage and scarring. In recent years, there have been an increasing number of reports of endophthalmitis caused by drug-resistant bacteria [9-11]. Therefore, novel, alternative, or additional treatments for antimicrobial-resistant bacteria are required.

As an application of phages to eye diseases, we previously reported the effectiveness of phage eyedrops against Pseudomonas aeruginosa keratitis in mice [12]. Additionally, the effectiveness of intravitreal injection of phages for vancomycin-sensitive and resistant enterococcal endophthalmitis in mice has been demonstrated [4]. Since enterococcal endophthalmitis exacerbates rapidly and the prognosis is poor, the development of novel therapeutic agents with prompt efficacy and broad host range, including antimicrobialresistant strains, is required. This study aimed to investigate the therapeutic potential of three newly isolated enterococcal phages on enterococcal endophthalmitis.

\section{Materials and Methods}

\subsection{Bacterial Strain Reagents and Culture Media}

Thirty non-vancomycin-resistant E. faecalis (VRE) strains, EF1-EF30, were isolated at the Clinical Laboratory Center in Kochi Medical School Hospital, Kochi, Japan, between 15 October and 20 December 1999, as described previously [13]. These 30 non-VRE strains were isolated from patients in different departments, had different antimicrobial sensitivities, and were isolated within a short period of time. Considering these factors, it is unlikely that they are the same strain. E. faecalis strains GU01-GU05 were isolated from four patients with postoperative endophthalmitis related to cataract surgery from four different hospitals, as described previously [4]. Four VRE strains, VRE1-VRE4, which were isolated independently, were kindly donated by Hokushin General Hospital, Nagano City, Nagano Prefecture, Japan [14]. E. faecalis EF24 was used as a host indicator strain for phiEF24C. E. faecalis strains GU01, GU02, and GU03 were used for animal experiments. All chemicals and reagents were obtained from Nacalai Tesque (Kyoto, Japan), Wako Chemicals (Osaka, Japan), or Sigma-Aldrich (St. Louis, MO, USA), unless otherwise stated. Heart infusion broth (HIB), brain heart infusion (BHI), and tyrosine soy broth (TSB) (Becton, Dickinson and Company, Franklin Lakes, NJ, USA) were used for bacterial and phage culture. Enterococcus Faecalis (EF) agar base (Nissui Pharmaceutical, Tokyo, Japan) was used to count colony forming units (CFUs) of EF24. Double-layered agar based on BHI medium, with $1.5 \%$ and $0.5 \%$ agar as the lower and the upper layers respectively, was used for evaluation of phage plaque formation.

\subsection{Phage Isolation}

A water sample was filtered through $0.45 \mu \mathrm{m}$ pore surfactant-free cellulose acetate membranes (Minisart; Sartorius Corporate Administration $\mathrm{GmbH}$, Goettingen, Germany), and $5 \mathrm{~mL}$ of the filtrate was mixed with $5 \mathrm{~mL}$ of $2 \times \mathrm{HIB}$ medium. The mixture was inoculated with $0.1 \mathrm{~mL}$ of overnight-cultured non-VRE strain and incubated at $37{ }^{\circ} \mathrm{C}$ overnight. The culture was filtered through a $0.45 \mu \mathrm{m}$ pore size membrane. Single plaque isolation was performed at least three times on the appropriate strains.

\subsection{Large-Scale Culture and Purification of Phages phiEF7H, phiEF14H1, and phiEF19G}

Phages phiEF7H, phiEF14H1, and phiEF19G were amplified with E. faecalis strains EF7, EF14, and EF24, respectively. BHI medium was used for phage purification in animal experiments. After complete bacterial lysis, centrifugation $\left(10,000 \times g, 10 \mathrm{~min}, 4{ }^{\circ} \mathrm{C}\right)$ was 
performed to remove bacterial debris. After centrifugation, phage-containing supernatants were collected, and polyethylene glycol 6000 and $\mathrm{NaCl}$ were added to final concentrations of $10 \%$ and $0.5 \mathrm{M}$, respectively. The suspension was stored at $4{ }^{\circ} \mathrm{C}$ overnight, and a phage pellet was obtained by centrifugation $\left(10,000 \times g, 20 \mathrm{~min}, 4^{\circ} \mathrm{C}\right)$, suspended in TM buffer (10 mM Tris- $\mathrm{HCl}(\mathrm{pH} 7.2)$ and $5 \mathrm{mM} \mathrm{MgCl} 2)$ containing DNase I $(100 \mu \mathrm{g} / \mathrm{mL})$ and RNase A $(100 \mu \mathrm{g} / \mathrm{mL})$, and incubated at $37^{\circ} \mathrm{C}$ for $30 \mathrm{~min}$.

For animal experiments, the crude phage suspension was layered on top of a discontinuous gradient of $40 \%, 35 \%$, and 30\% iodixanol (Opti-Prep; Alere Technologies, Oslo, Norway) in physiological saline and was ultracentrifuged $\left(50,000 \times g, 2 \mathrm{~h}, 4^{\circ} \mathrm{C}\right.$ or $100,000 \times g, 1 \mathrm{~h}, 4^{\circ} \mathrm{C}$ ) [4]. The phage band was collected and stored at $4{ }^{\circ} \mathrm{C}$ until use. Phage concentration in the plaque-forming unit (PFU) was measured using a plaque assay.

TSB medium was used for electron microscopic observation and DNA preparation. The crude phage suspension was layered on a discontinuous gradient $\rho=1.7, \rho=1.5$, $\rho=1.3 \mathrm{CsCl}$ in AAS $\left(100 \mathrm{mM}\right.$ ammonium acetate, $10 \mathrm{mM} \mathrm{NaCl}, 1 \mathrm{mM} \mathrm{MgCl} 2,1 \mathrm{mM} \mathrm{CaCl}_{2}$, ( $\mathrm{pH} 7.2)$ ) as described elsewhere [13]. After ultracentrifugation $\left(50,000 \times g, 2 \mathrm{~h}, 4{ }^{\circ} \mathrm{C}\right)$, the phage band was collected and dialyzed against $1 \mathrm{~L} \mathrm{AAS}$ for $1 \mathrm{~h}$ at $4{ }^{\circ} \mathrm{C}$.

\subsection{Measurement of Phage Host Range}

The host range of the three isolated phages was determined using the streak test. Briefly, phages were streaked using a $10 \mu \mathrm{L}$-loop on TSB-based double-layered agar plates inoculated with $E$. faecalis. After incubation $\left(24 \mathrm{~h}, 37^{\circ} \mathrm{C}\right)$, we recorded whether the bacteria had been lysed, lysed from without, or not lysed. Lysis from without was determined based on the appearance of a short transparent line with no plaque formation [15]. E. faecalis strain EF24 was used for the preparation of phage phiEF24. The titer of the phages phiEF7H, phiEF14H1, and phiEF19G and the phiEF24C stock was $15.7 \times 10^{9}, 14.1 \times 10^{9}, 11.8 \times 10^{9}$, and $10.2 \times 10^{9} \mathrm{CFU} / \mathrm{mL}$, respectively.

\subsection{Electron Microscopy}

Purified phages were placed on a mesh (Excel support film, Nisshin EM, Tokyo, Japan), and then negatively stained with $2 \%$ uranyl acetate ( $\mathrm{pH} 4.0)$. The samples were observed with a transmission electron microscope (JEOL JEM-1400Plus, JEOL, Tokyo, Japan) at $80 \mathrm{kV}$.

\subsection{Genome Sequencing and Analysis}

The DNA from the phages was prepared from the purified phage particles using previously described procedures [13]. Phage phiEF14H1 DNA was sequenced using the Illumina HiSeq2500 (Illumina, San Diego, CA, USA), and the reads were assembled using SPAdes assembler software v. 3. 10. One after trimming by Trimmomatic v. 0.36 [16]. DNA of phages phiEF7H and phiEF19G was sequenced using a GS Junior 454 sequencer (Roche Diagnostics, Risch-Rotkreuz, Switzerland). Sequence reads were assembled using 454 Newbler software (version 3.0; 454 Life Sciences, Branford, CT, USA) [17]. Genomes were annotated using a prokaryotic genome annotation pipeline, DFAST [18]. Genome sequences of phages phiEF14H1, phiEF7H, and phiEF19G were deposited in GenBank (accession numbers LC596378, LC596377, and LC596379, respectively).

To analyze the phylogenetic relationship of phages, the genome sequence data were analyzed using VICTOR with the default setting of formulas $\mathrm{d}_{0}$ [19]. Genome sequences were collected from GenBank (Table 1). 
Table 1. Genome sequences of phages belonging to family Herelleviridae genus Kochikohdavirus.

\begin{tabular}{ccccc}
\hline Phage Name & Length (bp) & Accession No. & Isolation & REF. \\
\hline phiEF7H & 143,399 & LC596377 & Japan & This study \\
phiEF14H1 & 143,280 & LC596378 & Japan & This study \\
phiEF19G & 143,400 & LC596379 & Japan & This study \\
phiEF17H & 143,638 & AP018714.1 & Japan & {$[20]$} \\
ECP3 & 145,518 & KJ801817.1 & Korea & [21] \\
PBEF129 & 144,230 & MN854830.1 & Korea & Unpublished \\
vB_EfaH_EF1TV & 143,507 & MK268686.1 & Italy & {$[22]$} \\
phiM1EF22 & 143,046 & AP018715.1 & Japan & [20] \\
vB_EfaM_Ef2.3 & 147,289 & MK721192.1 & USA & Unpublished \\
vB_EfaM_Ef2.1 & 140,938 & MK693030.1 & USA & Unpublished \\
phiEF24C & 142,072 & AP009390.1 & Japan & {$[13]$} \\
156 & 141,133 & LR031359.1 & Spain & {$[23]$} \\
EFLK1 & 130,952 & NC_029026.1 & Israel & [24] \\
\hline
\end{tabular}

\subsection{Mouse Model of Endophthalmitis}

\subsubsection{Ethical Treatment of Animals}

This study was approved by the Committee for Care and Use of Laboratory Animals at Kochi University (permit number M-00064) and was performed in accordance with the Association for Research in Vision and Ophthalmology (ARVO) Statement on the Use of Animals in Ophthalmic and Vision Research and with institutional guidelines for animal research.

\subsubsection{Mouse Model of Endophthalmitis}

Seven-week-old specific pathogen-free female C57BL/6J mice were obtained from Charles River Laboratories (Kanagawa, Japan) and housed under specific pathogen-free conditions at the animal facility of Kochi Medical School. E. faecalis strains GU01, GU02, GU03, and VRE2 were grown in $10 \mathrm{~mL} \mathrm{BHI}$ broth at $37^{\circ} \mathrm{C}$ to the logarithmic phase ( 100 Klett units, as measured with a Biowave CO8000 cell density meter (Biochrom, Cambridge, UK), and were then isolated by centrifugation at $10,000 \times g$ for 5 min at $4{ }^{\circ} \mathrm{C}$. The cell pellet was suspended in $10 \mathrm{~mL}$ physiological saline, and the suspension was centrifuged again under the same conditions. Cells were then suspended in $\sim 1 \mathrm{~mL}$ saline, and after appropriate dilution, turbidity (in Klett units) was measured to determine the bacterial cell number. One Klett unit was assumed to be equivalent to $7.9 \times 10^{6}$ E. faecalis cells $/ \mathrm{mL}$ on the basis of standardization, with bacterial cell numbers counted directly with a Petroff-Hausser counting chamber (Hausser Scientific, Horsham, PA, USA). A mouse model of endophthalmitis was constructed as previously described [4]. In brief, endophthalmitis of the right eye was induced by injection of $0.5 \mu \mathrm{L}$ of physiological saline containing $1 \times 10^{4}$ E. faecalis strains GU01, GU02, GU03, or VRE2 into the vitreous with a 36gauge needle. The left eye of each mouse was left untreated. At $6 \mathrm{~h}$ after bacterial injection, $1 \times 10^{8}$ PFU of phages phiEF7H, phiEF19G, or phiEF14H1 in $0.5 \mu \mathrm{L}$ of physiological saline, or $0.5 \mu \mathrm{L}$ of saline alone, were administered into the vitreous of the right eye.

\subsubsection{Measurement of Viable Bacteria in the Eye}

Eyes isolated $24 \mathrm{~h}$ after infection were disrupted to release bacteria in $1.0 \mathrm{~mL}$ ice-cold physiological saline using a tissue homogenizer (Mixer Mill MM300; Qiagen, Venlo, The Netherlands) for $5 \mathrm{~min}$ at maximum speed. Portions of each homogenate were diluted with saline and plated on EF agar base for culture at $37^{\circ} \mathrm{C}$ for $48 \mathrm{~h}$.

\subsubsection{Assay of Myeloperoxidase (MPO) Activity}

The number of neutrophils in the eye was estimated by measuring MPO activity as described previously, with slight modification. Briefly, eyes isolated $24 \mathrm{~h}$ post-infection were homogenized in $1.0 \mathrm{~mL}$ phosphate-buffered saline, the homogenate was centrifuged $\left(10,000 \times g, 15 \mathrm{~min}, 4^{\circ} \mathrm{C}\right)$, the resulting pellet was suspended in $0.03 \mathrm{~mL}$ of $50 \mathrm{mM}$ potassium 
phosphate buffer ( $\mathrm{pH}$ 6.0) containing $50 \mathrm{mM}$ hexadecyltrimethylammonium bromide, and $0.07 \mathrm{~mL}$ of $50 \mathrm{mM}$ potassium phosphate ( $\mathrm{pH}$ 6.0) was then added to the suspension. Each sample was subjected to three freeze-thaw cycles and then centrifuged at $10,000 \times g$ for $10 \mathrm{~min}$ at $4{ }^{\circ} \mathrm{C}$, after which $0.02 \mathrm{~mL}$ of the resulting supernatant was added to $0.05 \mathrm{~mL}$ of substrate reagent (R\&D Systems, Minneapolis, MN, USA), and the mixture was incubated for $20 \mathrm{~min}$ at room temperature. The reaction was terminated by adding $25 \mu \mathrm{L}$ of $2 \mathrm{~N}$ $\mathrm{H}_{2} \mathrm{SO}_{4}$, and the absorbance at $450 \mathrm{~nm}$ was measured.

\subsection{Statistics}

Quantitative data are presented as the mean \pm standard error of the mean (SEM) and were analyzed with Student's unpaired $t$-test. Statistical analysis was performed with statistical software (Statcel 4 software, OMS Publishing Inc., Saitama, Japan). A $p$-value of $<0.05$ was considered statistically significant.

\section{Results}

\subsection{In Vitro Effects of Phages in Bacteriolysis}

Phages were isolated from water samples collected from water channels in Kochi City, Kochi Prefecture, Japan. Three phages with broad host ranges were selected: phiEF7H, phiEF14H1, and phiEF19G, and their host range compared with that of the previously reported phage phiEF24C using 39 E. faecalis clinical isolates, including five endophthalmitisderived strains and four VRE strains. As shown in Table 2, 88.6\% of E. faecalis strains and $100 \%$ of VRE strains were lysed by phiEF19G and phiEF14H1. phiEF7H lysed $94.3 \%$ of $E$. faecalis strains and $100 \%$ of VRE strains. All endophthalmitis-derived clinical isolates were lysed by phiEF7H, phiEF14H1, and phiEF19G. However, only $80 \%$ of E. faecalis strains and $75 \%$ of VRE strains were lysed by phiEF24C.

\subsection{Characterization of Phages phiEF7H, phiEF14H1, and phiEF19G}

Phages phiEF7H, phiEF14H1, and phiEF19G were characterized from a morphological and phylogenetical point of view. First, observing these phages with transmission electron microscopy revealed an icosahedral head and a long contractile sheathed tail (Figure 1). The morphologies of these phages were generally similar in size and shape to the reported Enterococcus phage phiEF24C. In some electron micrographs of phage phiEF14H1 at 100,000 $\times$ magnification, a tail fiber with a bulging tip (ca. $83 \mathrm{~nm}$ in length), which protruded from the base plate of the tail, was often observed (white arrow in Figure 1C), as seen in the reported Enterococcus phage phiEF24C [13,15].

By analyzing the phylogenetic relationship to other related Enterococcus phages, the whole genomes of the phages phiEF7H, phiEF14H1, and phiEF19G were sequenced. The genomes of these phages contained 143,399, 143,280, and 143,400 bp, respectively. Nucleotide Basic Local Alignment Search Tool (BLASTn) analysis showed that each phage had $89 \%$ query coverage to phage phiEF24C in phages belonging to the Herelleviridae genus Kochikohdavirus. According to the phylogenetic analysis among the phages belonging to this virus taxonomy, phages phiEF7H, phiEF14H1, and phiEF19G were phylogenetically related to each other (Figure 2). Although these phages were isolated independently, they may be variants of the same phage strain because they are genetically and morphologically very similar to each other. However, although the deduced amino acid sequences of the tail fiber proteins (Gp31) of these three phages [15] were identical to each other, they were slightly different from that of phiEF24C (amino acid sequence identity of 98\%). This difference may be the cause of the slight difference in their host ranges. Thus, phages phiEF7H, phiEF14H1, and phiEF19G were classified into the Herelleviridae genus Kochikohdavirus. Since no lysogenic, toxic, or drug resistance gene disadvantageous to phage therapy was detected in the genomes of these phages, these phages were considered eligible as therapeutic phages to treat eye infections. 
Table 2. E. faecalis strains and their sensitivity to phages.

\begin{tabular}{|c|c|c|c|c|c|}
\hline \multirow{2}{*}{ Host } & \multirow{2}{*}{ Isolated Source } & \multicolumn{4}{|c|}{ Phage } \\
\hline & & phiEF7H & phiEF14H1 & phiEF19G & phiEF24C \\
\hline EF1 & Vaginal discharge & + & + & + & + \\
\hline EF2 & Vaginal discharge & + & + & + & + \\
\hline EF3 & Sputum & + & + & + & + \\
\hline EF4 & Pharyngis & + & + & + & LFW \\
\hline EF5 & Skin & + & + & + & + \\
\hline EF6 & Urine & + & + & + & + \\
\hline EF7 & Eye discharge & + & + & + & + \\
\hline EF8 & Other & + & + & + & + \\
\hline EF9 & Urine & - & - & - & + \\
\hline EF10 & Other & + & + & + & - \\
\hline EF11 & Urine & + & + & + & LFW \\
\hline EF12 & Urine & + & + & + & - \\
\hline EF13 & Pharyngis & - & - & - & - \\
\hline EF14 & Vaginal discharge & + & + & + & + \\
\hline EF15 & Pus & + & + & + & - \\
\hline EF16 & Vaginal discharge & + & + & + & + \\
\hline EF17 & Vaginal discharge & + & + & + & + \\
\hline EF18 & Vaginal discharge & + & + & + & + \\
\hline EF19 & Vaginal discharge & + & + & + & + \\
\hline EF20 & Pus & LFW & - & - & + \\
\hline EF21 & Pus & + & + & + & + \\
\hline EF22 & Mouthwash & + & + & + & + \\
\hline EF23 & Sputum & + & + & + & + \\
\hline EF24 & Vaginal discharge & + & + & + & + \\
\hline EF25 & Other & + & + & + & + \\
\hline EF26 & Vaginal discharge & + & + & + & + \\
\hline EF27 & Eye discharge & LFW & - & - & + \\
\hline EF28 & Vaginal discharge & + & + & + & + \\
\hline EF29 & Pus & + & + & + & + \\
\hline EF30 & Vaginal discharge & + & + & + & - \\
\hline GU01 & Endopthalmitis & + & + & + & + \\
\hline GU02 & Endopthalmitis & + & + & + & LFW \\
\hline GU03 & Endopthalmitis & + & + & + & LFW \\
\hline GU04 & Endopthalmitis & + & + & + & - \\
\hline GU05 & Endopthalmitis & + & + & + & - \\
\hline VRE1 & $\mathrm{HGH}$ & + & + & + & - \\
\hline VRE2 & $\mathrm{HGH}$ & + & + & + & + \\
\hline VRE3 & $\mathrm{HGH}$ & + & + & + & + \\
\hline VRE4 & $\mathrm{HGH}$ & + & + & + & + \\
\hline
\end{tabular}

+, plaque formation; -, no plaque formation; LFW, lysis from without. HGH, donated from Hokushin General Hospital, Nagano City, Japan.

\subsection{Effects of Intravitreous Phages on Bacterial Load in E. faecalis Endophthalmitis}

The effects of intravitreously administered phage phiEF7H, phiEF14H1, or phiEF19G were examined in a mouse model of E. faecalis endophthalmitis. Severe endophthalmitis was caused by the injection of $1 \times 10^{4}$ E. faecalis strains GU01, GU02, GU03, or VRE2 into the vitreous body. The ocular fundus was not visible because of fibrin precipitation and hemorrhage in the anterior chamber of vehicle-treated infected eyes observed by macroscopic examination. Intravitreous injection of phages $\left(1 \times 10^{8} \mathrm{PFU}\right)$ at $6 \mathrm{~h}$ after $E$. faecalis injection resulted in improvement of intracameral and intraocular inflammation at $24 \mathrm{~h}$, without fibrin and hemorrhage in the anterior chamber and the ocular fundus (Figures 3-5). 

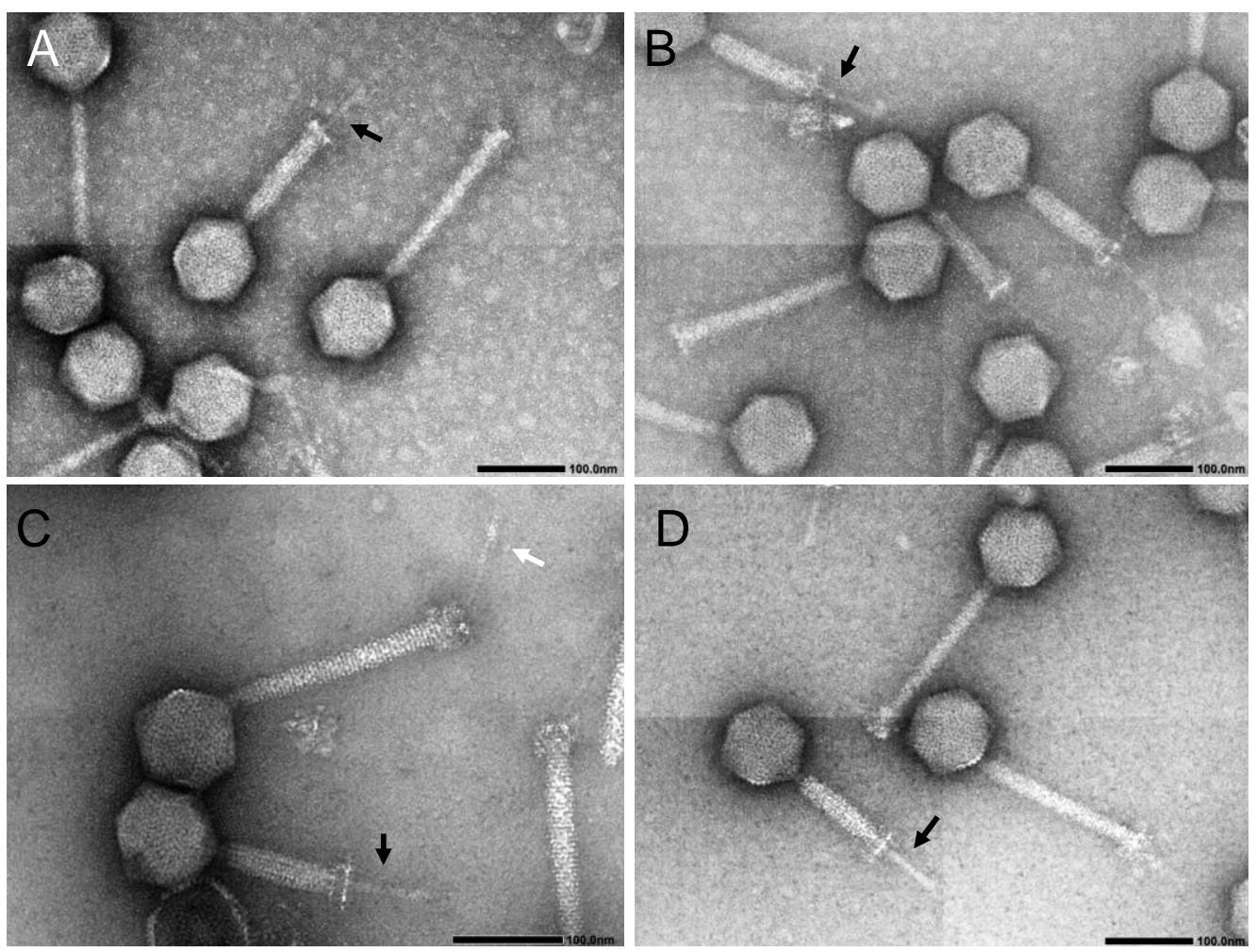

Figure 1. Transmission electron micrographs of negatively stained phage virions. (A) phiEF7H, (B,C) phiEF14H1, (D) phiEF19G. Bar, $100 \mathrm{~nm}$. The magnification of $(\mathbf{A}, \mathbf{B}, \mathbf{D})$ is $\times 80,000$ while that of (C) is $\times 100,000$. Black arrows in (A-D) indicate tail tube that appeared after the sheath contracted. White arrow in $(\mathrm{C})$ indicates a tail fiber protruding from the base plate of the tail. The size of each part is represented by the mean and standard deviation (mean $\pm \mathrm{SD}$ ) of the 10 samples, which were measured based on photographs with a magnification of $\times 30,000$. The head diameter was $101.5 \pm 3.9 \mathrm{~nm}$, the tail length was $220.3 \pm 4.6$, and the tail width was $21.6 \pm 1.5 \mathrm{~nm}$ in phiEF7H. The head diameter was $101.0 \pm 3.4 \mathrm{~nm}$, the tail length was $220.5 \pm 3.5$, and the tail width was $20.8 \pm 1.3 \mathrm{~nm}$ in phiEF14H1. The head diameter was $97.8 \pm 3.0 \mathrm{~nm}$, the tail length was $219.8 \pm 4.8$, and the tail width was $20.5 \pm 2.2 \mathrm{~nm}$ in phiEF19G.

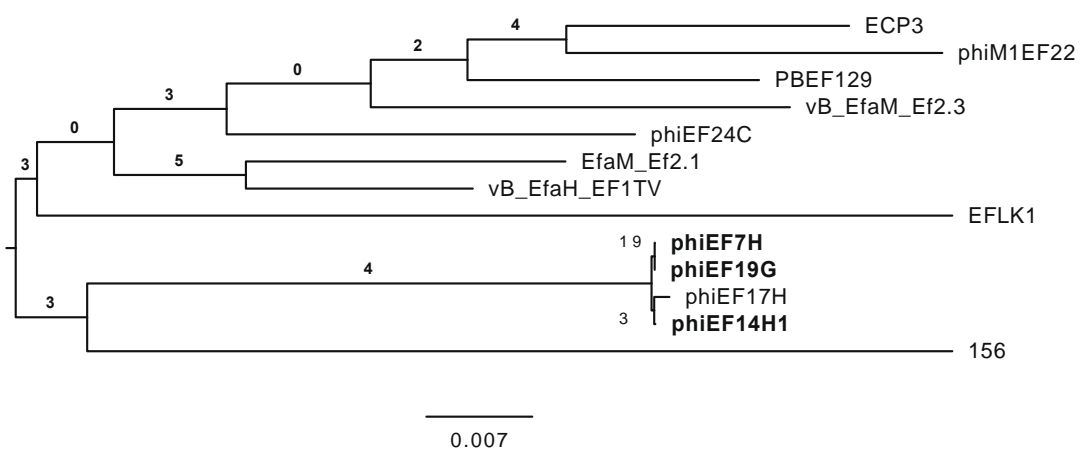

Figure 2. Phylogenetic tree among phages belonging to family Herelleviridae genus Kochikohdavirus. The phylogenetic tree was drawn using VICTOR [19]. The isolated phages phiEF7H, phiEF14H1, and phiEF19G are shown in bold. 

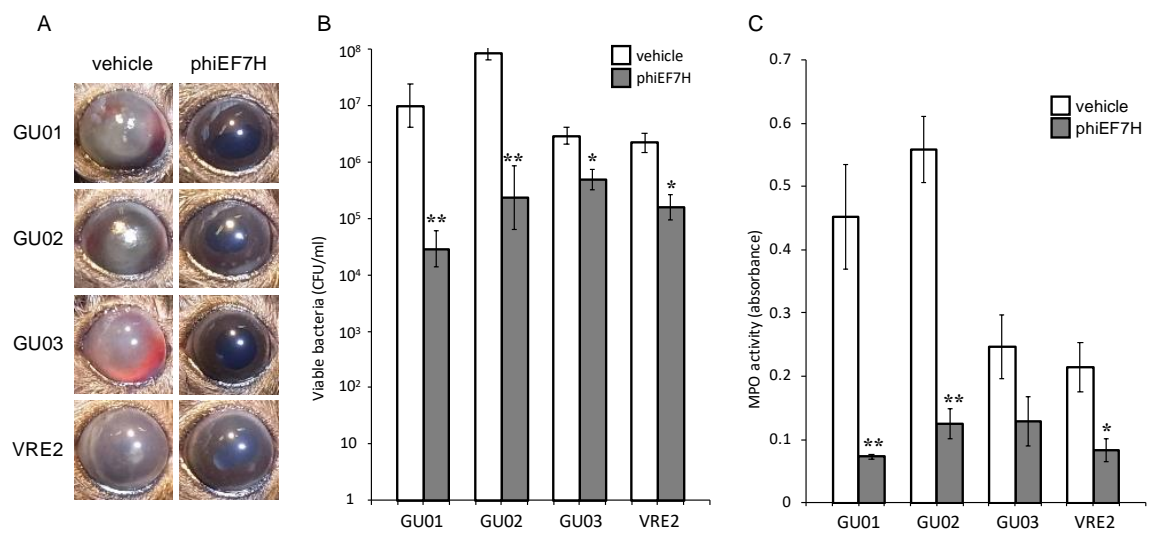

Figure 3. Effects of intravitreous injection of phage phiEF7H on live bacterial load and MPO activity for eyes with various E. faecalis-induced endophthalmitis. Eyes were injected with vehicle or phiEF7H at $6 \mathrm{~h}$ after injection of E. faecalis strain GU01, GU02, GU03, or VRE2. The clinical signs (A), viable bacterial load (B), and MPO activity (C) were determined $24 \mathrm{~h}$ after infection. All data are the mean \pm SEM for four to six eyes in each group. ${ }^{*}, p<0.05 ;{ }^{* *}, p<0.01$ (Student's $t$-test) versus vehicle-treated eyes.
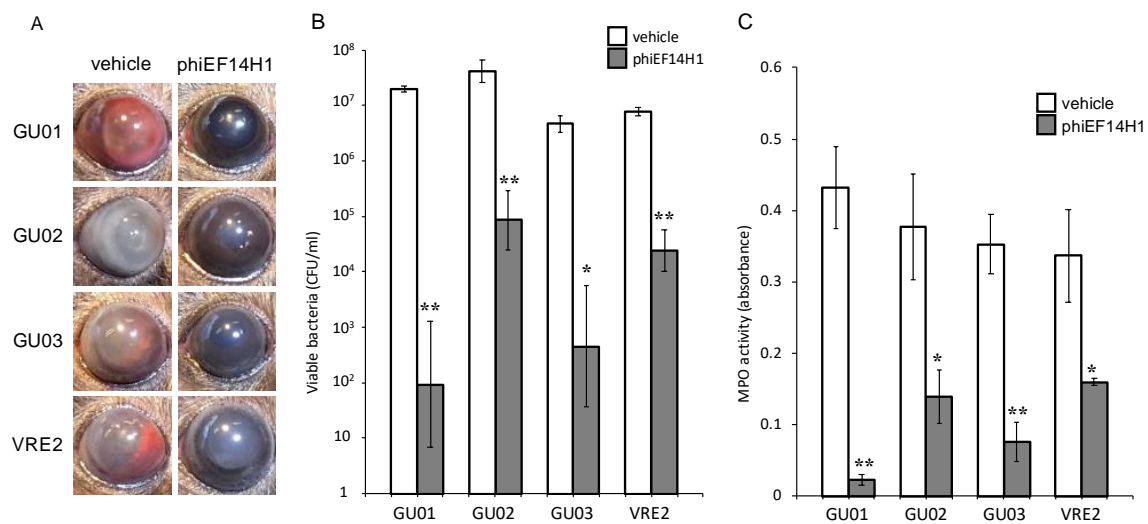

Figure 4. Effects of intravitreous injection of phage phiEF14H1 on live bacterial load and MPO activity for eyes with various E. faecalis-induced endophthalmitis. Eyes were injected with vehicle or phiEF14H1 at $6 \mathrm{~h}$ after injection of $E$. faecalis strain GU01, GU02, GU03, or VRE2. The clinical signs (A), viable bacterial load (B), and MPO activity (C) were determined $24 \mathrm{~h}$ after infection. All data are the mean \pm SEM for four to six eyes in each group. ${ }^{*}, p<0.05 ;{ }^{* *}, p<0.01$ (Student's $t$-test) versus vehicle-treated eyes.
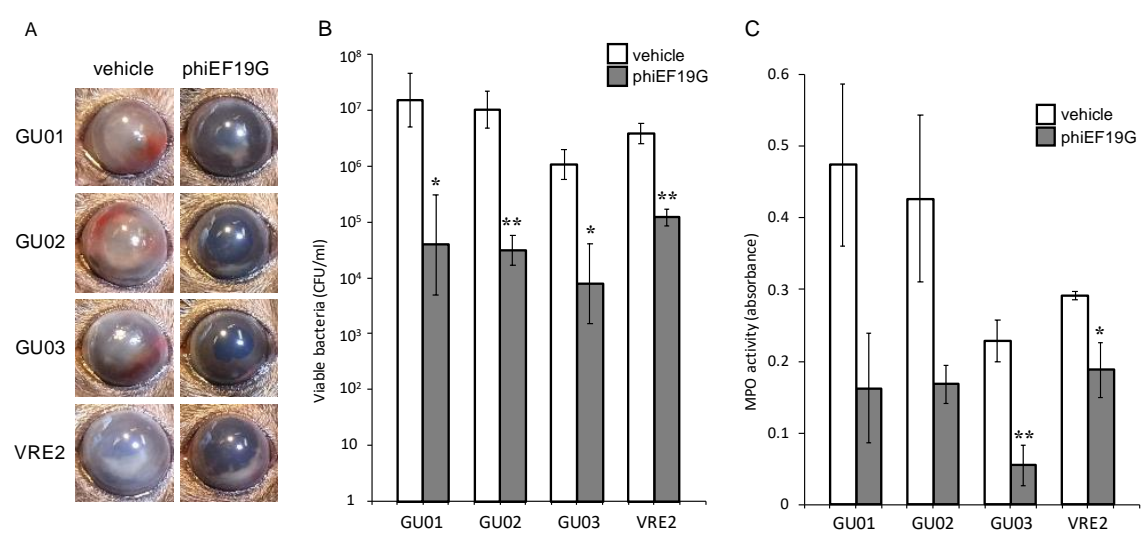

Figure 5. Effects of intravitreous injection of phage phiEF19G on live bacterial load and MPO activity for eyes with various E. faecalis-induced endophthalmitis. Eyes were injected with vehicle or phiEF19G at $6 \mathrm{~h}$ after injection of $E$. faecalis strain GU01, GU02, GU03, or VRE2. The clinical signs (A), viable bacterial load (B), and MPO activity (C) were determined $24 \mathrm{~h}$ after infection. All data are the mean \pm SEM for four to six eyes in each group. ${ }^{*}, p<0.05 ;{ }^{* *}, p<0.01$ (Student's $t$-test) versus vehicle-treated eyes. 
Eyeballs were assessed for bacterial load and MPO activity $24 \mathrm{~h}$ after intravitreal injection of E. faecalis. The live bacterial load in eyes injected with phages phiEF7H (Figure 3), phiEF19G (Figure 4), and phiEF14H1 (Figure 5) was significantly reduced compared with that of eyes injected with vehicle. Furthermore, MPO activity was decreased (Figures 3-5) compared with that of eyes inoculated with phages. The latter observation therefore suggested that infiltration of neutrophils into the eye was suppressed by intravitreal injection of phiEF7H, phiEF19G, and phiEF14H1.

\section{Discussion}

This study investigated the characteristics of three newly identified enterococcal phages. Based on the annotation using DNA Data Bank of Japan Fast Annotation and Submission Tool (DFAST), there were no genes associated with toxicity or pathogenicity or involved in the lysogenic life cycle. Moreover, the host spectrum of the isolated phages, phiEF7H, phiEF14H1, and phiEF19G, was much broader than that of previously reported phage phiEF24C [13] when these phages and phiEF24C were tested against $E$. faecalis strains isolated from clinical specimens (Table 2). In particular, we found that these three phages could lyse all five strains derived from endophthalmitis and four strains of VRE. Thus, the isolated phages, phiEF7H, phiEF19G, and phiEF14H1, were considered promising for the therapeutic purpose of endophthalmitis from the phage therapy point of view. Given that the host spectrum of these phages differed from that of phiEF24C, it is possible that a cocktail of these phages could be effective therapeutic agents for a broader range of enterococci.

Intravitreous administration of phage was recently reported to be effective against E. faecalis endophthalmitis in mice. Local phage therapy was shown to reduce the load of viable bacteria, suppress neutrophil infiltration, and protect the function of the retina [4]. Enterococcal endophthalmitis progresses quickly and requires prompt treatment. We have previously shown that phage induced lysis of E. faecalis in vitro faster than vancomycin in vitro, and thus, intravitreous phage therapy is a potential candidate for treating endophthalmitis [4]. Phages phiEF7H, phiEF14H1, and phiEF19G reduced the load of viable bacteria and suppressed neutrophil infiltration in a mouse model of endophthalmitis caused by VRE and E. faecalis isolated from endophthalmitis. The multiplication of phages depends on the growth of host bacteria; therefore, it is important to confirm the therapeutic effects in vitro and in vivo. This study demonstrated that phages phiEF7H, phiEF14H1, and phiEF19G lyse VRE and E. faecalis isolated from endophthalmitis in vitro and in vivo and thus can be considered novel therapeutic agent candidates. Phages with a broader host spectrum, such as these three, may be effective as new therapeutic agents for endophthalmitis caused by both antimicrobial-sensitive and resistant bacteria.

Ocular infectious diseases caused by antimicrobial-resistant bacteria are reportedly increasing and are becoming a clinical issue that requires addressing. Antimicrobial-resistant strains of various types of bacteria derived from ocular infection have been isolated, including S. aureus, coagulase-negative staphylococci, P. aeruginosa, Corynebacterium spp., and Escherichia coli [25-29]. In recent years, novel alternative or additional therapeutic agents to antibiotics have been developed for ocular infections caused by antimicrobial-resistant bacteria, including antiseptic hexamidine diisethionate ophthalmic solution [30] and devices to improve the antibiotic bioavailability of eye drops such as polyvinyl alcohol/anionic collagen membranes [31] and ofloxacin-loaded polymeric nanoparticles [32]. We have previously reported the effectiveness of phage eye drops for the mouse model of $P$. aeruginosa keratitis [12] and others have reported a case with vancomycin-resistant $S$. aureus keratitis successfully treated by a bacteriophage eyedrop [33]. Phages can be applied to various dosage forms, such as eye drops, ointments, or vitreous injection, and serve as potential candidates for treating drug-resistant bacterial infections of the eye.

Correct delivery of phages to the focus of infection is important for successful phage therapy. The ability to deliver phages directly and accurately by eye drops, intracameral injection, or intravitreous injection is an advantage for the treatment and prophylaxis of 
ocular infectious diseases. For clinical application, it is necessary to confirm the safety of the eye and the pharmacokinetic excretion pathway after intraocular administration of phages. We have previously confirmed that intravitreously injected phages remained in the eye for at least 3 days and had no toxic effects on retinal function at 2 weeks after injection in mice [4]. Postoperative endophthalmitis progresses rapidly, but the incidence is low; however, it is important to develop safe prophylaxis for this condition. Recently, antimicrobial agents are often administered by eye drops or intracameral injections at the end of surgery for the prophylaxis of postoperative endophthalmitis. However, cases of severe hemorrhagic occlusive retinal vasculitis after intracameral injection of vancomycin that causes visual loss have been reported [34,35]. Therefore, new therapeutic agents are required to replace antimicrobial agents for the prophylaxis of postoperative endophthalmitis. Given that postoperative endophthalmitis usually occurs within a few days after intraocular surgeries, phages that can remain in the eye for at least several days may also be suitable for prophylaxis. It is necessary to investigate the safety and accurate pharmacokinetic excretion pathway in the eyes with respect to the phages phiEF7H, phiEF14H1, and phiEF19G in future studies. As described above, these three phages, which may be variants of the same phage strain, showed similar therapeutic effects on eye infections, strongly suggesting that phages that belong to the same derivative group in the Kochikohdavirus genus may be eligible as therapeutic phages for eye infections.

\section{Conclusions}

Three newly isolated enterococcal phages were studied that could lyse broad-range E. faecalis, including strains derived from endophthalmitis and VRE in vitro and in vivo. Thus, these isolated phages may be promising candidates for phage therapy against endophthalmitis.

Author Contributions: Conceptualization, K.F. and S.M.; data curation, T.K., W.I. and K.F.; formal analysis, T.K., W.I., T.N., J.U. and S.M.; methodology, W.I., T.N., T.U. and T.S.; investigation, T.K., W.I., T.N., T.U., I.N., S.M. and K.F.; resources, J.U., D.T. and S.M.; funding acquisition, K.F.; writingoriginal draft preparation, T.K., T.N. and K.F.; writing-review and editing, T.S., J.U., D.T., M.D. and A.F.; supervision, K.F.; project administration, K.F. All authors have read and agreed to the published version of the manuscript.

Funding: This research was supported by Japan Agency for Medical Research and Development (AMED) and Japan Science and Technology Agency (JST).

Conflicts of Interest: J.U., M.D., A.F., S.M. and K.F. are the inventors of patents of these phages.

\section{References}

1. Matsuzaki, S.; Uchiyama, J.; Takemura-Uchiyama, I.; Daibata, M. Perspective: The age of the phage. Nature 2014, 509, S9. [CrossRef] [PubMed]

2. Watanabe, R.; Matsumoto, T.; Sano, G.; Ishii, Y.; Tateda, K.; Sumiyama, Y.; Uchiyama, J.; Sakurai, S.; Matsuzaki, S.; Imai, S.; et al. Efficacy of bacteriophage therapy against gut-derived sepsis caused by Pseudomonas aeruginosa in mice. Antimicrob. Agents Chemother. 2007, 51, 446-452. [CrossRef] [PubMed]

3. Takemura-Uchiyama, I.; Uchiyama, J.; Osanai, M.; Morimoto, N.; Asagiri, T.; Ujihara, T.; Daibata, M.; Sugiura, T.; Matsuzaki, S. Experimental phage therapy against lethal lung-derived septicemia caused by Staphylococcus aureus in mice. Microbes Infect. 2014, 16, 512-517. [CrossRef] [PubMed]

4. Kishimoto, T.; Ishida, W.; Fukuda, K.; Nakajima, I.; Suzuki, T.; Uchiyama, J.; Matsuzaki, S.; Todokoro, D.; Daibata, M.; Fukushima, A. Therapeutic Effects of Intravitreously Administered Bacteriophage in a Mouse Model of Endophthalmitis Caused by Vancomycin-Sensitive or -Resistant Enterococcus faecalis. Antimicrob. Agents Chemother. 2019, 63. [CrossRef]

5. West, E.S.; Behrens, A.; McDonnell, P.J.; Tielsch, J.M.; Schein, O.D. The incidence of endophthalmitis after cataract surgery among the U.S. Medicare population increased between 1994 and 2001. Ophthalmology 2005, 112, 1388-1394. [CrossRef]

6. Pershing, S.; Lum, F.; Hsu, S.; Kelly, S.; Chiang, M.F.; Rich, W.L., 3rd; Parke, D.W., 2nd. Endophthalmitis after Cataract Surgery in the United States: A Report from the Intelligent Research in Sight Registry, 2013-2017. Ophthalmology 2020, 127, 151-158. [CrossRef]

7. The Endophthalmitis Vitrectomy Study Group. Microbiologic factors and visual outcome in the endophthalmitis vitrectomy study. Am. J. Ophthalmol. 1996, 122, 830-846. [CrossRef]

8. Todokoro, D.; Suzuki, T.; Kobayakawa, S.; Tomita, H.; Ohashi, Y.; Akiyama, H. Postoperative Enterococcus faecalis endophthalmitis: Virulence factors leading to poor visual outcome. Jpn. J. Ophthalmol. 2017, 61, 408-414. [CrossRef] 
9. Babalola, O.E. Intravitreal linezolid in the management of vancomycin-resistant enterococcal endophthalmitis. Am. J. Ophthalmol. Case Rep. 2020, 20, 100974. [CrossRef]

10. Bains, H.S.; Weinberg, D.V.; Feder, R.S.; Noskin, G.A. Postoperative vancomycin-resistant Enterococcus faecium endophthalmitis. Arch. Ophthalmol. 2007, 125, 1292-1293. [CrossRef]

11. Sharma, S.; Desai, R.U.; Pass, A.B.; Saffra, N.A. Vancomycin-resistant enterococcal endophthalmitis. Arch. Ophthalmol. 2010, 128, 794-795. [CrossRef] [PubMed]

12. Fukuda, K.; Ishida, W.; Uchiyama, J.; Rashel, M.; Kato, S.; Morita, T.; Muraoka, A.; Sumi, T.; Matsuzaki, S.; Daibata, M.; et al. Pseudomonas aeruginosa keratitis in mice: Effects of topical bacteriophage KPP12 administration. PLoS ONE 2012, 7, e47742. [CrossRef] [PubMed]

13. Uchiyama, J.; Rashel, M.; Maeda, Y.; Takemura, I.; Sugihara, S.; Akechi, K.; Muraoka, A.; Wakiguchi, H.; Matsuzaki, S. Isolation and characterization of a novel Enterococcus faecalis bacteriophage phiEF24C as a therapeutic candidate. FEMS Microbiol. Lett. 2008, 278, 200-206. [CrossRef] [PubMed]

14. Oana, K.; Kawakami, Y.; Ohnishi, M.; Ishikawa, M.; Hirota, M.; Tozuka, M.; Atarashi, K.; Baba, K.; Fujiki, K.; Okazaki, M.; et al. Molecular and epidemiological study of the first outbreak of vanB type vancomycin-resistant Enterococcus faecalis in Japan. Jpn. J. Infect. Dis. 2001, 54, 17-22.

15. Uchiyama, J.; Takemura, I.; Satoh, M.; Kato, S.; Ujihara, T.; Akechi, K.; Matsuzaki, S.; Daibata, M. Improved adsorption of an Enterococcus faecalis bacteriophage PhiEF24C with a spontaneous point mutation. PLoS ONE 2011, 6, e26648. [CrossRef]

16. Bolger, A.M.; Lohse, M.; Usadel, B. Trimmomatic: A flexible trimmer for Illumina sequence data. Bioinformatics 2014, 30, 2114-2120. [CrossRef]

17. Silva, G.G.; Dutilh, B.E.; Matthews, T.D.; Elkins, K.; Schmieder, R.; Dinsdale, E.A.; Edwards, R.A. Combining de novo and reference-guided assembly with scaffold_builder. Source Code Biol. Med. 2013, 8, 23. [CrossRef]

18. Tanizawa, Y.; Fujisawa, T.; Nakamura, Y. DFAST: A flexible prokaryotic genome annotation pipeline for faster genome publication. Bioinformatics 2018, 34, 1037-1039. [CrossRef]

19. Meier-Kolthoff, J.P.; Goker, M. VICTOR: Genome-based phylogeny and classification of prokaryotic viruses. Bioinformatics 2017, 33, 3396-3404. [CrossRef]

20. Uchiyama, J.; Matsui, H.; Murakami, H.; Kato, S.I.; Watanabe, N.; Nasukawa, T.; Mizukami, K.; Ogata, M.; Sakaguchi, M.; Matsuzaki, S.; et al. Potential Application of Bacteriophages in Enrichment Culture for Improved Prenatal Streptococcus agalactiae Screening. Viruses 2018, 10, 552. [CrossRef]

21. Kang, H.; Kim, S.; Kim, J. Isolation and characterization of an Enterococcus faecalis bacteriophage. Korean J. Microbiol. 2015, 51, 194-198. [CrossRef]

22. D'Andrea, M.M.; Frezza, D.; Romano, E.; Marmo, P.; Henrici De Angelis, L.; Perini, N.; Thaller, M.C.; Di Lallo, G. The lytic bacteriophage vB_EfaH_EF1TV, a new member of the Herelleviridae family, disrupts biofilm produced by Enterococcus faecalis clinical strains. J. Glob. Antimicrob. Resist. 2020, 21, 68-75. [CrossRef] [PubMed]

23. Del Rio, B.; Sanchez-Llana, E.; Redruello, B.; Magadan, A.H.; Fernandez, M.; Martin, M.C.; Ladero, V.; Alvarez, M.A. Enterococcus faecalis Bacteriophage 156 Is an Effective Biotechnological Tool for Reducing the Presence of Tyramine and Putrescine in an Experimental Cheese Model. Front. Microbiol. 2019, 10, 566. [CrossRef] [PubMed]

24. Khalifa, L.; Coppenhagen-Glazer, S.; Shlezinger, M.; Kott-Gutkowski, M.; Adini, O.; Beyth, N.; Hazan, R. Complete Genome Sequence of Enterococcus Bacteriophage EFLK1. Genome Announc. 2015, 3. [CrossRef] [PubMed]

25. Cabrera-Aguas, M.; Khoo, P.; George, C.R.R.; Lahra, M.M.; Watson, S.L. Antimicrobial resistance trends in bacterial keratitis over 5 years in Sydney, Australia. Clin. Exp. Ophthalmol. 2020, 48, 183-191. [CrossRef]

26. Parchand, S.M.; Agrawal, D.; Chatterjee, S.; Gangwe, A.; Mishra, M.; Agrawal, D. Post-cataract surgery cluster endophthalmitis due to multidrug-resistant Pseudomonas aeruginosa: A retrospective cohort study of six clusters. Indian J. Ophthalmol. 2020, 68, 1424-1431. [CrossRef]

27. Khan, M.; Stapleton, F.; Summers, S.; Rice, S.A.; Willcox, M.D.P. Antibiotic Resistance Characteristics of Pseudomonas aeruginosa Isolated from Keratitis in Australia and India. Antibiotics 2020, 9, 600. [CrossRef]

28. Alexandrakis, G.; Alfonso, E.C.; Miller, D. Shifting trends in bacterial keratitis in south Florida and emerging resistance to fluoroquinolones. Ophthalmology 2000, 107, 1497-1502. [CrossRef]

29. Ranjith, K.; SaiAbhilash, C.R.; Sai Prashanthi, G.; Padakandla, S.R.; Sharma, S.; Shivaji, S. Phylogenetic Grouping of Human Ocular Escherichia coli Based on Whole-Genome Sequence Analysis. Microorganisms 2020, 8, 422. [CrossRef]

30. Pinna, A.; Donadu, M.G.; Usai, D.; Dore, S.; Boscia, F.; Zanetti, S. In Vitro Antimicrobial Activity of a New Ophthalmic Solution Containing Hexamidine Diisethionate 0.05\% (Keratosept). Cornea 2020, 39, 1415-1418. [CrossRef]

31. Daza, J.H.U.; Righetto, G.M.; Chaud, M.V.; da Conceicao Amaro Martins, V.; Lopes Baratella da Cunha Camargo, I.; Maria de Guzzi Plepis, A. PVA/anionic collagen membranes as drug carriers of ciprofloxacin hydrochloride with sustained antibacterial activity and potential use in the treatment of ulcerative keratitis. J. Biomater. Appl. 2020, 35, 301-312. [CrossRef] [PubMed]

32. Salama, A.H.; AbouSamra, M.M.; Awad, G.E.A.; Mansy, S.S. Promising bioadhesive ofloxacin-loaded polymeric nanoparticles for the treatment of ocular inflammation: Formulation and in vivo evaluation. Drug Deliv. Transl. Res. 2020. [CrossRef] [PubMed]

33. Fadlallah, A.; Chelala, E.; Legeais, J.M. Corneal Infection Therapy with Topical Bacteriophage Administration. Open Ophthalmol. J. 2015, 9, 167-168. [CrossRef] [PubMed] 
34. Witkin, A.J.; Shah, A.R.; Engstrom, R.E.; Kron-Gray, M.M.; Baumal, C.R.; Johnson, M.W.; Witkin, D.I.; Leung, J.; Albini, T.A.; Moshfeghi, A.A.; et al. Postoperative hemorrhagic occlusive retinal vasculitis: Expanding the clinical spectrum and possible association with vancomycin. Ophthalmology 2015, 122, 1438-1451. [CrossRef] [PubMed]

35. Todorich, B.; Faia, L.J.; Thanos, A.; Amin, M.; Folberg, R.; Wolfe, J.D.; Todorich, K.M.; Raphtis, E.; Ruby, A.J.; Williams, G.A.; et al. Vancomycin-associated hemorrhagic occlusive retinal vasculitis: A clinical-pthophysiological analysis. Am. J. Ophthalmol. 2018, 188, 131-140. [CrossRef] 